\title{
Official clinical practice guideline: Mechanical ventilation in adult patients with acute respiratory distress syndrome
}

\author{
Kenneth Nugent MD, Hawa Edriss MD
}

The American Thoracic Society, the European Society of Intensive Care Medicine, and the Society of Critical Care Medicine recently released an official clinical practice guideline for mechanical ventilation in adult patients with acute respiratory distress syndrome (ARDS). ${ }^{1}$ This panel considered six important questions in patients with ARDS, reviewed the literature related to each question, summarized the literature related to each question, and made recommendations. This editorial will review these questions and recommendations.

Question 1: Should patients with ARDS receive mechanical ventilation using low tidal volumes and inspiratory pressures?

This panel recommended that adult patients with ARDS should receive mechanical ventilation with strategies that limit the tidal volume $(4-8 \mathrm{mls} / \mathrm{kg}$ predicted body weight) and inspiratory pressures (plateau pressure $<30 \mathrm{~cm} \mathrm{H}_{2} \mathrm{O}$ ). This was a strong recommendation with a moderate confidence in the effect estimate. It was based on nine randomized controlled trials which included 1,629 patients. However, the primary analysis of seven trials (without high PEEP levels per protocol) indicated that mortality was not significantly different in patients managed with a low tidal volume strategy compared to a traditional strategy (7 studies; 1,481 patients; risk ratio: $0.87,95 \%$ confidence interval: $0.7-1.08)$. This result seems peculiar since there is almost universal acceptance that a low tidal volumes strategy in these patients reduces mortality. However, meta-regression analysis showed that there was an inverse relationship between larger tidal volume gradients (i.e., the differences between the higher tidal

Corresponding author: Kenneth Nugent Contact Information: Kenneth.nugent@ttuhsc.edu DOI: 10.12746/swrccc.v5i20.411 volume in control patients and the low tidal volume in experimental patients) and the relative risk for mortality associated with low tidal volume ventilation; trials with larger gradients showed a lower mortality risk with low tidal volumes. In addition, an analysis which also included the trials with low tidal volumes and higher PEEP levels showed significantly reduced mortality (9 studies; 1,629 patients; relative risk: 0.8 , 95\% confidence interval: $0.66-0.98)$. There was no difference in barotrauma (3 studies, 1,029 patients) or ventilator-free days (2 studies, 977 patients) in the articles reviewed for this question.

In summary, the best current recommendation is to use a low tidal volume strategy in patients with ARDS to reduce ventilator-induced lung injury. The panel recommended an increase in the tidal volume to $8 \mathrm{ml} / \mathrm{kg}$ predicted body weight if patients are double triggering the ventilator, or if the inspiratory airway pressure falls below the PEEP level. Recent data from randomized controlled trials suggested that the driving pressure $(\triangle P=$ plateau pressure-PEEP) is a better predictor of ARDS outcomes than tidal volume or plateau pressures. Further controlled trials are needed to study the effect of driving pressure in predicting lung injury. ${ }^{2}$

Question 2: Should patients with ARDS receive prone positioning?

The panel recommended that adult patients with severe ARDS should receive prone positioning for more than 12 hours per day. This was a strong recommendation with a moderate to high confidence in the effect estimate. It was based on eight randomized controlled trials which included 2,129 patients. Severe ARDS was defined as a $\mathrm{PaO}_{2} / \mathrm{FiO}_{2}$ ratio in the range of 100-150. Overall there was no difference in mortality in these trials when comparing patients in prone versus supine position. However, prespecified subgroup analysis indicated that the patients with moderate to severe ARDS placed in the prone position for more 
than 12 hours per day had improved survival. This mortality benefit was confirmed by the results of the PROSEVA trial (Proning Severe ARDS Patients) in 2013 which reported a significant survival benefit. ${ }^{3}$ Prone positioning is associated with increased endotracheal tube obstruction and more pressure sores.

In summary, patients with severe ARDS defined by a $\mathrm{PaO}_{2} / \mathrm{FiO}_{2}$ ratio less than 150 should have prone positioning for more than 12 hours per day. This increases the delivery of tidal volume to the lung bases which leads to more uniform gas distribution throughout the lungs, improves ventilation-perfusion relationships, and potentially decreases ventilatorinduced lung injury.

Question 3: Should patients with ARDS receive high-frequency oscillatory ventilation?

The panel recommended that high frequency oscillatory ventilation should not be used routinely in patients with moderate or severe ARDS. This was a strong recommendation with moderate to high confidence in the effect estimate. It was based on six randomized control trials which included 1,715 patients. There was no difference in mortality between patients receiving high-frequency oscillatory ventilation and patients in the control groups. One study reported a significantly higher mortality rate in the high-frequency oscillatory ventilation compared to patients treated with a low tidal volume strategy and higher PEEP levels. Two studies reported no differences in barotrauma.

In summary, high-frequency oscillatory ventilation should not be routinely used in patients with ARDS.

Question 4: Should patients with ARDS receive higher as compared to lower PEEP?

The panel suggested that adult patients with moderate to severe ARDS should receive higher rather than lower levels of PEEP. This was a conditional recommendation with moderate confidence in the effect estimate. They reviewed eight randomized controlled trials which included 2,728 patients. The mean high level PEEP was $15.1 \pm 3.6 \mathrm{~cm}$ of water versus $9.1 \pm$ $2.7 \mathrm{~cm}$ of water in the low PEEP group. There was no significant difference in mortality between patients on higher versus lower PEEP levels. Higher PEEP levels were not associated with significant differences in barotrauma, new organ failure, or ventilator free days. However, the panel's recommendation was based on evidence from three randomized controlled trials which provided individual patient level data for meta-analysis. In these studies, patients with moderate to severe ARDS defined by a $\mathrm{PaO}_{2} / \mathrm{FiO}_{2} \leq 200$ had significantly lower mortality rates if randomized to higher PEEP levels (relative risk $=0.9 ; 95 \% \mathrm{Cl}=0.81$ 1.0). However, the best method to set higher PEEP levels in these patients is unclear. Levels which increase the plateau pressure above $30 \mathrm{~cm}$ of $\mathrm{H}_{2} \mathrm{O}$ have the potential to cause more harm than good.

In summary, consider higher PEEP levels in patients with very abnormal oxygenation defined by $\mathrm{PaO}_{2} / \mathrm{FiO}_{2}$ ratios.

\section{Question 5: Should patients with ARDS receive recruitment maneuvers?}

This panel suggested that adult patients with ARDS should receive recruitment maneuvers. However, this was a conditional recommendation with low to moderate confidence in the effect estimate. Lung recruitment maneuvers instituted by a transient elevation in the airway pressures to open ("recruit") atelectatic lung regions with subsequent reductions in alveolar dead space, short term improvement in pulmonary compliance, and reduction in intrapulmonary shunting. This analysis was based on six randomized controlled trials which included 1,423 patients. The type of recruitment maneuver varied widely among these trials. Some trials included higher PEEP levels as a co-intervention. When considering all six trials, recruitment maneuvers were associated with significantly lower mortality rates (relative risk $=0.81 ; 95 \%$ confidence interval $=0.69-0.95$ ). These maneuvers are not associated with barotrauma or hemodynamic compromise. However, the optimal method for recruitment, the frequency of recruitment, and the target patient group for recruitment are unclear. Typically, these patients will require higher PEEP levels to maintain more "open" lungs following recruitment.

In summary, consider recruitment maneuvers with careful attention to changes oxygenation and the time frame for improvement. 
Question 6: Should patients with ARDS receive extracorporeal membrane oxygenation?

The panel did not make a recommendation regarding the use of ECMO in patients with severe ARDS. They noted that the most recent randomized controlled trials have significant limitations, including composite endpoints, incomplete application of the intervention, the lack of a standardized low tidal volume ventilation strategy in the control group, and transfer to high volume referral centers.

In summary, ECMO should be used only in high volume centers with ongoing clinical studies.

In summary, these guidelines recommend a low tidal volume and inspiratory pressure strategy in patients with ARDS. Patients with severe ARDS should receive prone positioning for at least 12 hours per day. Some patients with severe ARDS may benefit from higher PEEP levels, but the exact level is uncertain. Some patients may benefit from recruitment maneuvers, but the best approach to these maneuvers is uncertain. Finally, these patients should not receive high-frequency oscillatory ventilation or undergo extracorporeal membrane oxygenation except in clinical trials.
Keywords: acute respiratory distress syndrome, mechanical ventilation, low tidal volume, prone position, guideline

\section{Submitted: $7 / 10 / 2017$ \\ Conflicts of interest: none}

\section{REFERENCES}

1. Fan E, Del Sorbo L, Goligher EC, et al. An Official American Thoracic Society, the European Society of Intensive Care Medicine, and the Society of Critical Care Medicine. An Official American Thoracic Society/European Society of Intensive Care Medicine/Society of Critical Care Medicine Clinical Practice Guideline: Mechanical ventilation in adult patients with acute respiratory distress syndrome. Am J Respir Crit Care Med 2017 May 1;195(9):1253-1263.

2. Amato MBP, Meade MO, Arthur S, et al. Driving pressure and survival in the acute respiratory distress syndrome. N Engl $\mathrm{J}$ Med 2015;372:747-755.

3. Guérin C, Reignier J, Richard JC, Beuret P, Gacouin A, et al. PROSEVA Study Group. Prone positioning in severe acute respiratory distress syndrome. N Engl J Med. 2013 Jun 6;368(23):2159-68. 